in

n the first part of our review of cardiac development, ${ }^{1}$ we discussed the initial changes involved in transformation of the heart forming regions of the embryo into the great veins, the atrial and ventricular chambers, and the arterial trunks. We showed that this first part of cardiac development could be divided into phases of formation of the primary myocardial tube, looping of the tube, during which additional parts are added that give the future compartments their definitive topography, and the assembly of these components into the cardiac chambers and arterial trunks. In this second review, we discuss the processes that complete the separation of the two sides of the definitive heart, for the most part involving septation of the parts of the primary tube not themselves directly involved in formation of the chamber-specific compartments of the atriums and ventricles. In this respect, when concluding our first review, we explained how the arterial trunks were also formed by septation within the solitary outflow tract of the primary heart tube. We also showed, however, that subsequent to formation of the two arterial trunks, there was disappearance of the cushions that initially divided them. Thus, in the definitive heart, the proximal parts of the aorta and pulmonary trunk, along with the sinuses of the arterial roots and the subpulmonary infundibulum, possess their own discrete walls, separated by extra-cardiac space. Although septation by fusion of endocardial cushions will be a topic included in this second review, septation and separation of the outflow tracts is sufficiently complicated to require special treatment. Because of this, we will devote a third review specifically to the mechanisms underscoring the remodelling of the outflow tracts. In this review, therefore, we will confine our considerations to the formation of the atrial, atrioventricular, and ventricular septal structures.

\title{
MORPHOLOGICAL CONSIDERATIONS
}

See end of article for authors' affiliations

Correspondence to: Professor Robert H Anderson, Cardiac Unit, Institute of Child Health, 30 Guilford Street, London WC1H 1EJ, UK;

r.anderson@ich.ucl.ac.uk
As will become evident, separation of the four cardiac chambers involves more than simple formation of partitions between them. There is justification for arguing that understanding the complex mechanics of development itself will provide the information required properly to interpret the postnatal morphology. Clinicians, however, are more likely to be acquainted with the structure of the heart rather than its development. We will preface our account of the developmental events, therefore, with a brief review of the nature of the tissues that separate the cavities on the right and left sides in the definitive heart. Appreciation of the precise structure of these separating walls underpins the understanding of the developmental steps involved in their formation.

Thus, careful examination of the heart subsequent to separation of the chambers shows that several of the structures usually described as "septums" in classical accounts ${ }^{2}$ are, in reality, no more than folds, with extracardiac adipose tissue incorporated between the folded layers of myocardium. It can reasonably be argued, of course, that any partition interposed between the cavities of adjacent cardiac chambers is justifiably described as a septum, irrespective of its precise structure. Our own understanding was helped, nonetheless, when we came to appreciate the anatomic difference between shelves within the chambers, as opposed to those folded myocardial walls that incorporate between them wedges of extracardiac fibro-adipose tissue. ${ }^{3}$ This difference is best seen at the oval fossa ("fossa ovalis"). The flap valve, forming the floor of the fossa, and being free to open and close while the fossa is patent, is the exemplar of the shelf-like partition that we define as a true septal structure. ${ }^{3}$ The antero-inferior muscular buttress that anchors the flap valve to the fibrous atrioventricular septal structures is also a true septum, since it, too, can be removed without creating a communication with the extracardiac space. In contrast, the so-called "septum secundum", seen supero-posteriorly, is a deep infolding between the walls of the superior caval and right pulmonary veins at their junctions with their respective atriums (fig 1). Incisions in this area, initially extending into the subepicardial fibro-adipose tissue, can potentially extend to the pericardial cavity.

Yet another arrangement of overlapping tissues is to be found in the area of the triangle of Koch. In this area, which separates the off-set attachments of the tricuspid and mitral valvar leaflets, a layer of fibro-adipose tissue is again incorporated between two muscular layers, but in this example, the muscular layers - one atrial and the other ventricular-are both attached antero-superiorly 


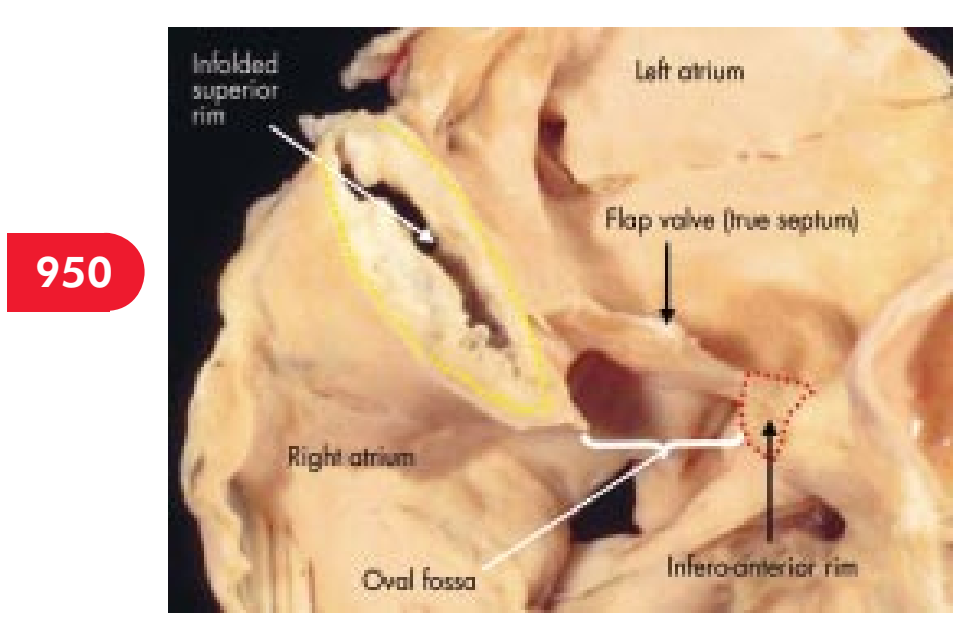

Figure 1 This adult human heart has been sectioned in "four chamber" plane to reveal the nature of the rims of the oval fossa (fossa ovalis). The floor of the fossa is the flap valve, which if removed would create a communication between right and left atriums. The infero-anterior rim (red dotted lines) is a muscular buttress that can also be removed to create a communication between the chambers. The extensive superior rim (yellow dotted lines), however, is a fold between the attachments of the caval veins to the right atrium and the pulmonary veins to the left atrium. Cutting through this rim would create a communication with the extracardiac space.

to the central fibrous body. The fibro-adipose layer between the two muscular walls is itself continuous with the inferior atrioventricular groove (fig 2). Although previously we nominated this area as a "muscular atrioventricular septum", ${ }^{4}$ septation itself cannot produce this arrangement. Rather, as we will describe, the folding occurs concomitant with caudal expansion of the right atrioventricular junction over the base of the ventricular mass.

The significance of morphologic distinctions of this type should become evident as we discuss the development of the various structures that, eventually, separate the atrial and ventricular chambers within the definitive heart. These are the atrial septum, the atrioventricular septum, and the ventricular septum. We will start, however, with a brief consideration of another area often described as a septum, again erroneously in our view, namely the tissues that separate the two horns of the systemic venous sinus ("sinus venosus").

\section{FORMATION OF THE "SEPTUM" OF THE SYSTEMIC VENOUS SINUS}

As described in our first review, ${ }^{1}$ the systemic venous tributaries exist from the outset of formation of the circulatory system, being continuous with the caudal, or atrial, end of the developing heart tube. These systemic venous tributaries are initially formed in symmetrical fashion in the right and left sides of the embryo. In the earliest human embryo that we have studied, nonetheless, at Carnegie stage 12, the veins are already joined to the primary atrial component of the heart tube in asymmetric fashion (fig 3). This is because, already at this early stage of development in the human, the left sinus horn is becoming incorporated into the developing left atrioventricular groove, passing beneath the posterior mesocardium. The mesocardium itself provides the portal for the pulmonary vein, when it develops, to enter the left atrium. ${ }^{15}$

An integral part of ongoing atrial development is reorientation of the openings of the systemic venous tributaries so that they empty only to the right side of the primary atrium, this
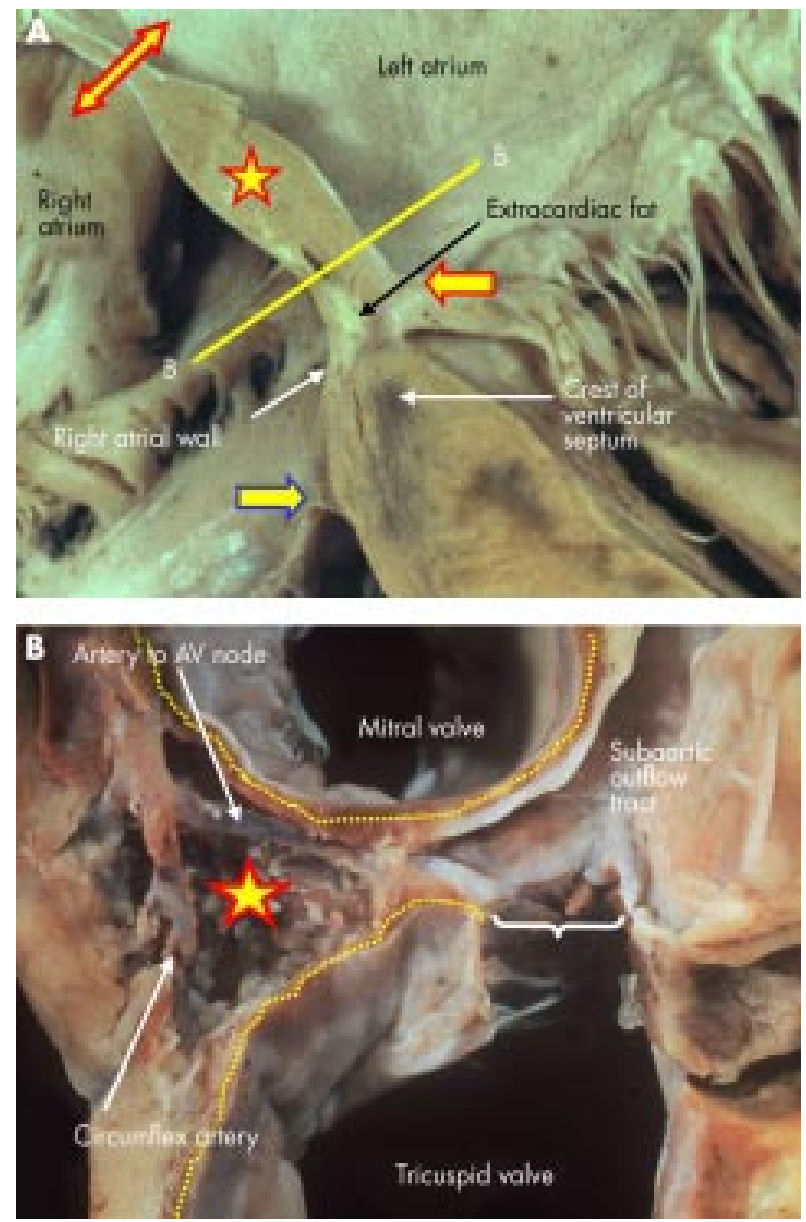

Figure 2 The upper panel $(A)$ is another "four-chamber" section through an adult human heart, this time taken closer to the crux. It shows the area of off-setting between the hinges of the tricuspid (blue and yellow arrow) and mitral (red and yellow arrow) valves. Initially, we described this area as a muscular "septum". As can be seen in the section, however, extracardiac adipose tissue is incorporated between the layers of atrial and ventricular musculature, producing a notably different anatomic arrangement separating the atrial cavities when compared to the flap valve (double headed arrow) or the muscular buttress (star) at the base of the flap valve. The lower panel (B) shows a view of the short axis of the cardiac mass along the plane $B-B$ in fig $2 A$, viewed from the atrial aspect. It shows the origin and extent of the extracardiac adipose tissue, which extends anteriorly and superiorly between the atrial and ventricular muscle masses from the inferior atrioventricular groove (star), with the artery to the atrioventricular node coursing through the tissue plane thus created. The yellow dotted lines show the cut edges of the atrial musculature, and the bracket shows the site of the membranous septum, which has been removed.

being a necessary prelude to atrial septation. Almost always, in the human, there is an accompanying refiguration of the venous pathways themselves. Anastomoses are formed in the neck and abdomen, permitting a greater volume of blood to be returned to the heart through the right sided tributaries, which persist at their junction with the atrium as the orifices of the superior and inferior caval veins. There is concomitant diminution in size of the left sinus horn, which persists as the coronary sinus, developing so as to receive most of the venous return from the heart itself. The left sinus horn is also smaller than the right horn in the mouse heart (fig 4A). In the mouse, however, the left sinus horn remains patent in postnatal life, draining the persisting left superior caval vein. Such a 


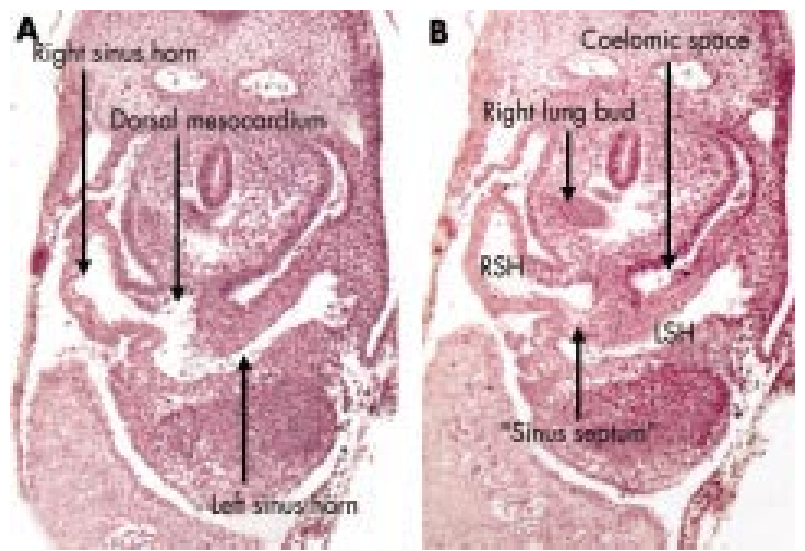

Figure 3 These sections in transverse plane across the embryo are from a human embryo measuring $4 \mathrm{~mm}$ from crown to rump, at the 12th stage according to the Carnegie classification. Panel A shows the right and left sinus horns at their attachment to the primary atrium, with the dorsal, or posterior, mesocardium connecting the primary atrium itself to the posterior wall of the embryo. At this stage, there is only minimal formation of the lung buds, with the right bud just visible in panel B. This panel, taken slightly more inferiorly compared to panel A, shows the fold between the two sinus horns (RSH, LSH) at their junction with the primary atrium. This is the so-called "septum" of the embryonic systemic venous sinus.

situation can also be found as a congenital malformation in the human, with the left caval vein maintaining both its patency and its connection to the right atrium. Reduction in size of the left horn, therefore, is not a pre-requisite for successful atrial septation. Be that as it may, it is the initial bifurcation of the walls of the right and left horns, at their entrances to the systemic venous sinus, that persists in the human and the mouse as the so-called "sinus septum" (fig 4B). This so-called septum, therefore, is no more than a muscular fold in the medial wall of the right atrium, interposing between the orifices of the inferior caval vein and the coronary sinus.

\section{FORMATION OF THE ATRIAL SEPTUM}

As we have described, the systemic venous tributaries are reorientated as a prelude to septation so as to enter exclusively the right side of the primary atrial component of the heart tube. And, concomitant with this reorientation of the systemic venous tributaries, the pulmonary vein develops as a new structure, using the persisting posterior mesocardium to gain its entrance to the left side of the primary atrium. ${ }^{56}$ Subsequent to these changes in venous topography, the scene is set for the initial primary atrial component of the heart tube to be divided into its own right and left components, each with an appendage ballooned from the convex parietal side of the atrial body. ${ }^{1}$ It is the distinctive structure of each appendage that confers morphological specificity on the new right and left atriums.

Septation is accomplished in several steps. Two endocardial cushions are produced within the initially common atrioventricular junction, also part of the primary heart tube (figs 5 and 6), while the primary atrial septum grows as a muscular partition from the roof of the primary atrial component of the heart tube (figs 6 and 7). This muscular shelf grows into the atrial cavity between the systemic and pulmonary venous openings (fig 8), taking its origin from the part of the primary atrium that has molecularly left characteristics, as shown by its expression of the Pitx2 gene. The part of the primary atrium with these left sided molecular characteristics extends as far
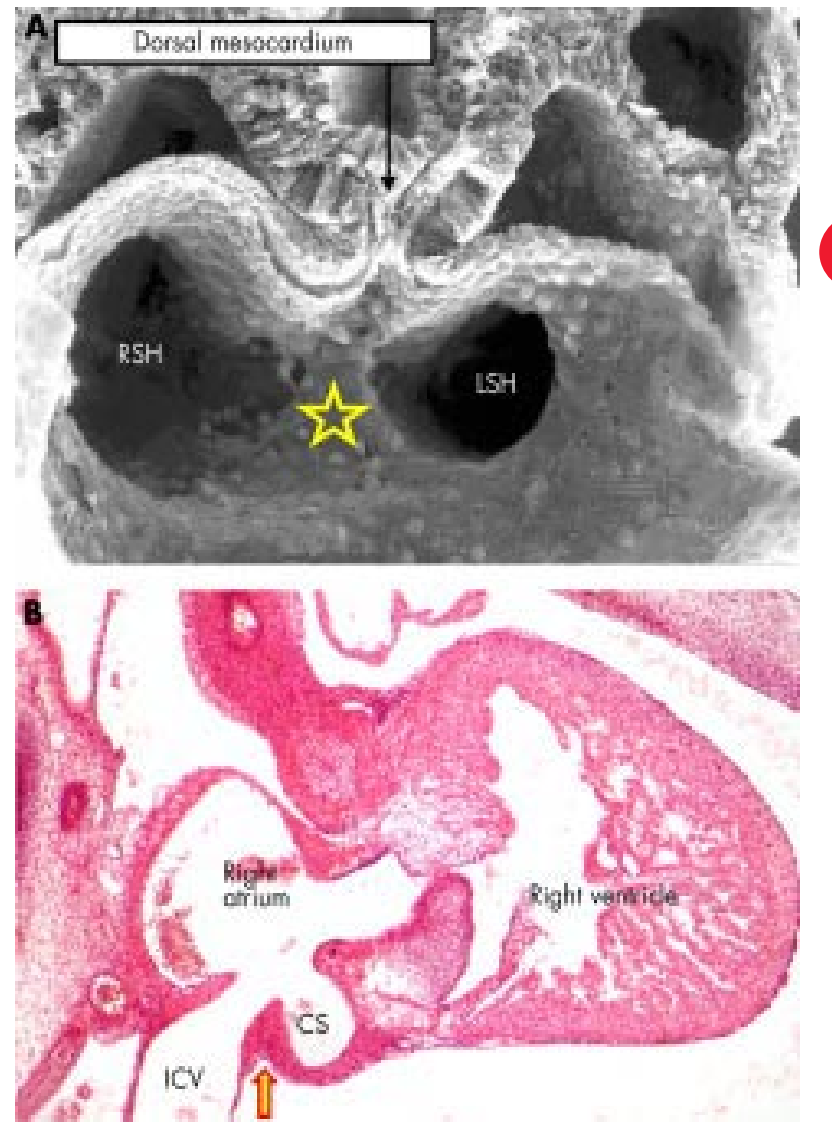

Figure 4 These panels show the fate of the so-called "septum" of the systemic venous sinus. Panel $A$ is from a mouse embryo on the 10 th day of development. The star shows the tissue separating the mouths of the right and left sinus horns at their junction with the primary atrium. Note the dorsal mesocardium, and compare with panel $B$ of fig 3. Panel B is from a human embryo at stage 20 of the Carnegie classification, sectioned more or less in parasternal long axis plane. It shows the fold between the entrance of the coronary sinus (CS), derived from the left sinus horn, and that of the inferior caval vein (ICV), formed from the lower end of the opening of the right sinus horn. This fold is the so-called "septum" of the systemic venous sinus.

rightwards as the attachments of the left valve of the systemic venous sinus. ${ }^{7}$ As the primary muscular septum grows from the atrial roof, with its origin leftward relative to the developing left venous valve, it carries a mesenchymal cap on its leading edge (fig 9). With continuing growth of the muscular primary atrial septum, this mesenchymal cap approaches, and then fuses with, the superior endocardial cushion of the atrioventricular canal (fig 10). Concomitant with this development, a further mass of mesenchyme (fig 11) grows into the heart inferiorly from the posterior mediastinum. ${ }^{8}$ At this stage, the pulmonary vein remains a solitary channel, which opens to the left atrium inferiorly, adjacent to the atrioventricular junction. The mass of mesenchyme entering the heart from the mediastinum, called the "spina vestibuli" by His, grows through the rightward border of the new pulmonary venous orifice, enlarging the border in the process (fig 8). As it grows into the atrium, this vestibular spine also carries forward the inferior ends of the so-called venous valves, which by now guard the orifices of the systemic venous sinus, marking their junction with the developing right atrium (fig 9). The process of merging and fusion of the mesenchymal cap on the 


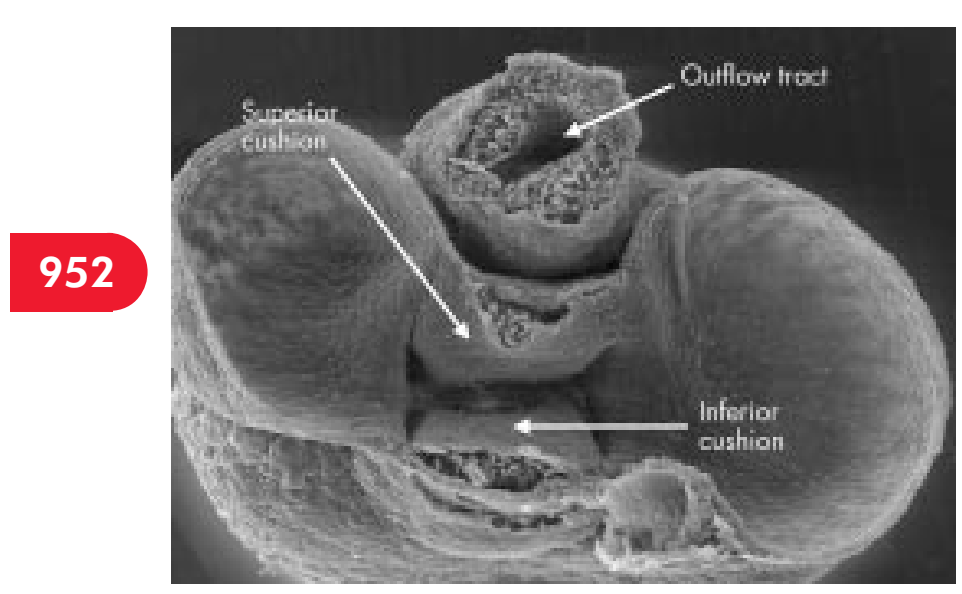

Figure 5 This picture shows the atrioventricular canal in a mouse heart during the 10th day of development, viewed from above having transected the developing heart tube. Note the cushions filling the common atrioventricular canal, and growing towards each other from the superior and inferior quadrants. Note also that the oufflow tract is a solitary tube at this stage, also with opposing cushions developing within its lumen.

primary septum with the mesenchymal tissues of the vestibular spine, combined with subsequent fusion with the superior and inferior atrioventricular cushions, themselves also fused by this stage, serves to fill in the gap initially seen beneath the leading edge of the primary septum and the atrioventricular endocardial cushions. In other words, it is the fusion of the various mesenchymal components that closes the primary atrial foramen, or "ostium primum". At the same time, the process of fusion divides the common atrioventricular junction into its right and left components. Before the primary atrial foramen is closed, however, the upper margin of the muscular primary septum breaks down to form the secondary interatrial foramen, or the "ostium secundum" (figs 6 and 7). After fusion of the various mesenchymal structures, the primary septum itself is firmly adherent, via the tissues derived from the vestibular spine and the mesenchymal structures, to the crest of the ventricular septum. This permits the primary septum itself to function as the flap valve of the oval foramen. The mass of tissue derived from the vestibular spine now forms an extensive buttress at the right side of the base of the primary septum. This tissue then muscularises, except for the central part continuous posteriorly and inferiorly with the valves of the systemic venous sinus. This remains as the fibrous tendon of Todaro. The muscularised component forms a true secondary atrial septum at the base of the flap valve, the latter, of course, representing the persisting muscular primary atrial septum (fig 12).

When first formed, the secondary foramen has no upper rim, its border being the flat atrial roof (fig 10). Only much later, subsequent to separation of the right and left pulmonary veins, and incorporation of their orifices as separate openings to the roof of the left atrium, does the deep fold develop to form the structure usually described as the "septum secundum". It is against this fold that the flap valve abuts in postnatal life to close the oval foramen (fig 12).

\section{FORMATION OF THE ATRIOVENTRICULAR SEPTUM}

As explained above, before growth of the primary atrial septum from the roof of the atrium, two masses of mesenchymal tissue had formed superiorly and inferiorly within the

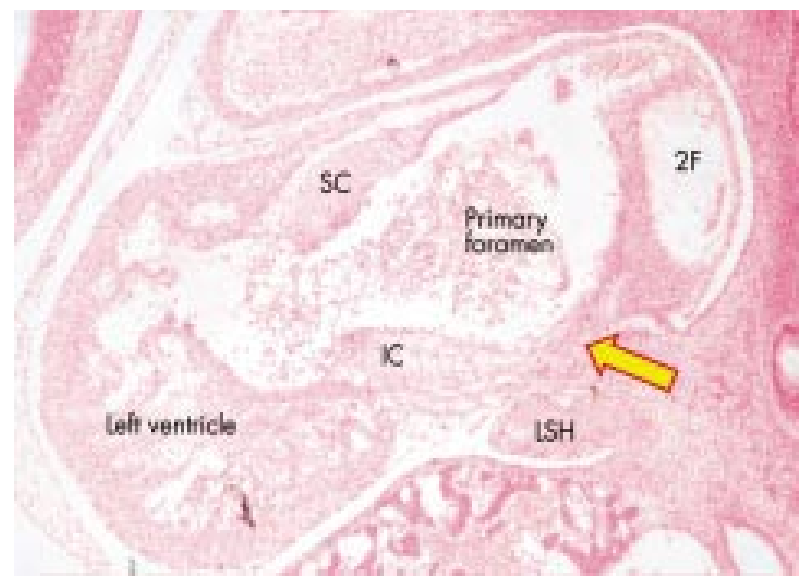

Figure 6 This section, in parasternal long axis plane, is from a human embryo at stage 14 of the Carnegie classification. The endocardial cushions (SC, IC) have formed superiorly and inferiorly within the atrioventricular canal, which at this stage has appreciable length. The left sinus horn (LSH) is now incorporated as a channel with its own walls within the developing left atrioventricular junction. The primary septum itself has grown down from the atrial roof, but has broken down superiorly to produce the secondary interatrial foramen (2F). The primary foramen is seen between the primary septum and the as yet unfused atrioventricular cushions. The section shows also the window for growth into the heart from the posterior mediastinum (yellow and red arrow). This is the vestibular spine.

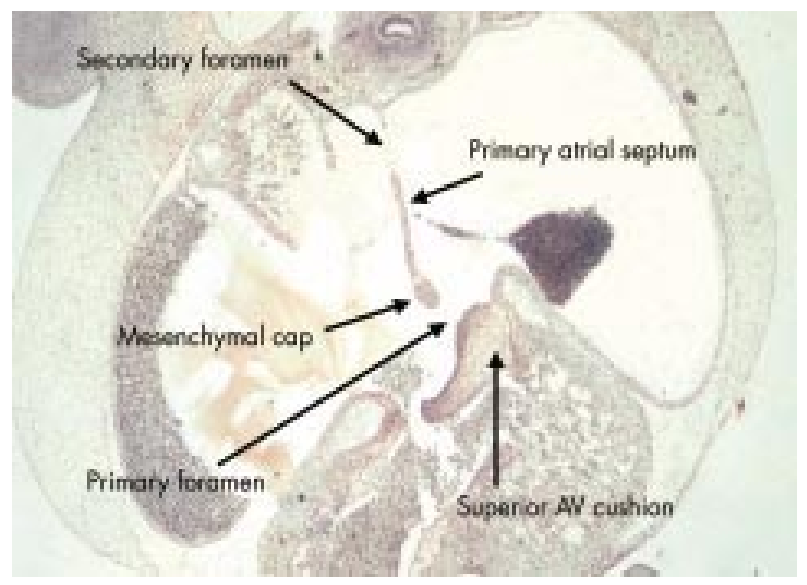

Figure 7 This section, in "four chamber" plane, is from a human embryo at stage 16 of the Carnegie classification. The section has passed through the superior cushion of the atrioventricular (AV) canal, which has yet to fuse with its partner. The primary atrial septum has grown down from the atrial roof, carrying a mesenchymal cap on its leading edge. Note, however, that the upper edge of the primary septum has already broken down to form the secondary interatrial foramen. The primary foramen is still patent, between the mesenchymal cap and the atrial surface of the atrioventricular cushions.

atrioventricular canal. These are the atrioventricular endocardial cushions (figs 5 and 6). The two cushions grow towards each other, fusing to divide the canal into its right and left components (fig 10). The cushions are also adherent to the crest of the muscular ventricular septum, this septum itself forming as the apical trabecular components of the developing left and right ventricles balloon from the primary heart tube (see below). A key feature in growth of this primary ventricular septum, however, is the increase in size of its posteroinferior component. This part of the muscular septum develops concomitant with growth of the inlet of the right 


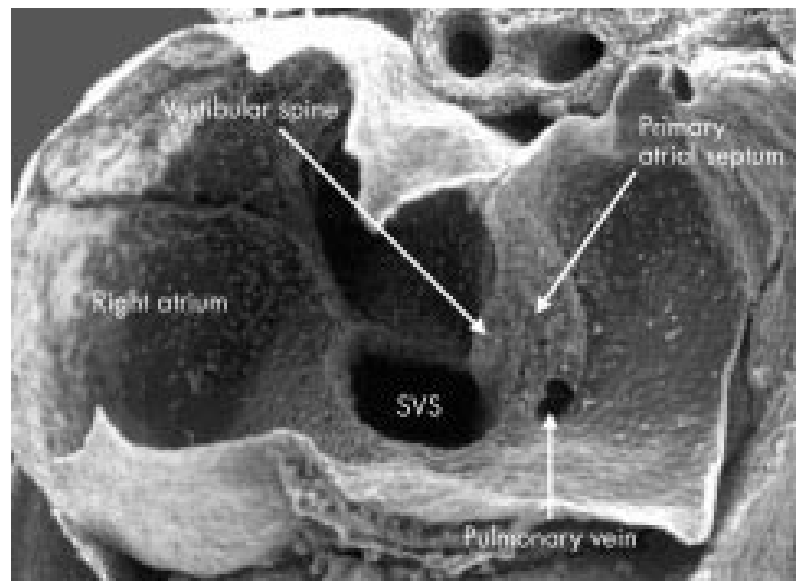

Figure 8 This scanning electron micrograph is from a mouse heart at a slightly earlier stage of development than the human embryo shown in fig 6. The atrioventricular canal has been dissected away from the atrial chambers, permitting the atrial cavities to be photographed from beneath. The valves of the systemic venous sinus have now formed, showing that the sinus itself (SVS) opens exclusively to the right side of the primary atrium. Note that the orifice of the pulmonary vein now opens inferiorly to the left side of the atrium. The primary atrial septum is growing as a shelf between the systemic and pulmonary venous orifices. Note also the bulge at the right side of the pulmonary venous orifice, between it and the opening of the systemic venous sinus. This is the "spina vestibuli" initially described by His, ${ }^{9}$ which we term the vestibular spine.

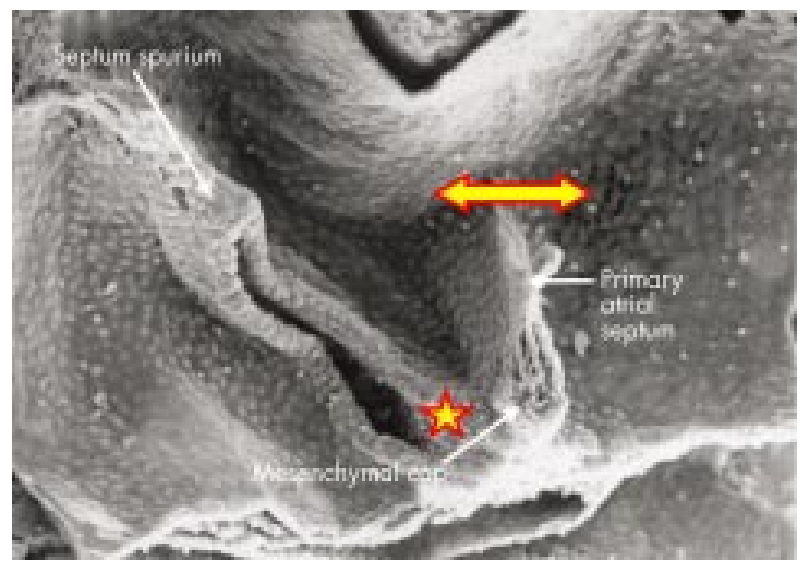

Figure 9 This higher power scanning electron micrograph shows the atrial roof, prepared in comparable fashion to fig 8 , but at a slightly later stage of development. The upper margin of the primary atrial foramen has now broken down to form the secondary interatrial foramen (double headed arrow). Note the mesenchymal cap on the leading edge of the primary septum, which is growing towards the atrioventricular endocardial cushions, carrying forward the inferior end of the orifice of the systemic venous sinus (star). The upper end of the orifice becomes the septum spurium in the roof of the right atrial appendage.

ventricle, this self evidently occurring subsequent to division of the atrioventricular canal. Having divided, the atrioventricular canal expands rightward and, as it does so, the larger part of the inferior atrioventricular cushion becomes draped over the right side of the enlarging postero-inferior part of the muscular ventricular septum. The larger part of the superior cushion, in contrast, together with a smaller part of the inferior cushion at the site of fusion, remains within the cavity of the developing left ventricle. The line of fusion with the inferior cushion then forms the supero-posterior margin of the

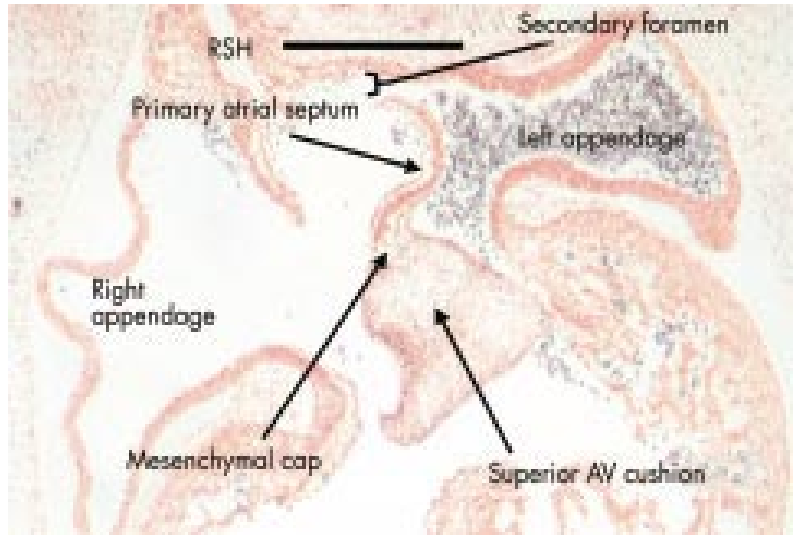

Figure 10 This section, in four chamber plane, is from a human embryo at stage 16 of the Carnegie classification. It shows how the mesenchymal cap on the primary atrial septum has now fused with the superior cushion, with breakdown of the upper edge to form the secondary foramen. The upper margin of the foramen, however, is flat to the orifice of the right sinus horn ( $\mathrm{RSH}$, black line). Only subsequent to septation is there infolding of the superior margin (see fig 12).

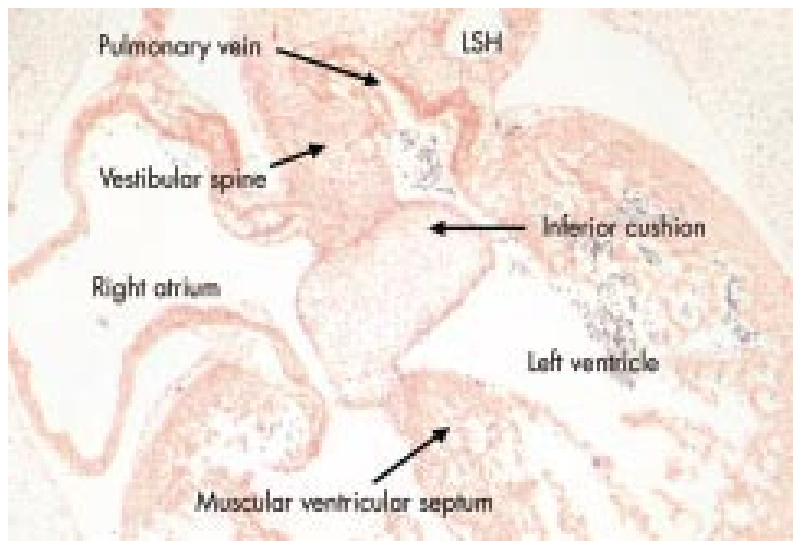

Figure 11 This section, in four chamber plane, is from the same human embryo at stage 16 of the Carnegie classification as shown in fig 10. This section shows the inferior cushion adherent to the ventricular septum, but with the larger part of its bulk within the left ventricle. It also shows the tissue growing into the heart through the vestibular spine, confining the pulmonary vein within the developing left atrium. Note the left sinus horn (LSH) with its own discrete walls.

embryonic interventricular foramen. This part of the foramen has now become an arch-like structure that, eventually, will provide the vestibule of the subaortic part of the left ventricular outflow tract (fig 13).

The tricuspid orifice itself has increased in size concomitant with the expansion of the atrioventricular canal, and growth of the inlet part of the right ventricle. The mitral orifice also increases in size. It is only at a much later stage, after the completion of ventricular septation (see below), that the musculature of the initial atrioventricular canal that surrounded the developing valvar orifices, apart from the area destined to become the atrioventricular conduction axis, loses its continuity with the ventricular myocardium. This separation is produced by formation of rings of fibro-adipose tissue throughout the newly expanded atrioventricular junctions. This process of separation, which produces the essential plane of electrical atrioventricular insulation, occurs at a level that sequestrates the musculature of the initial atrioventricular 


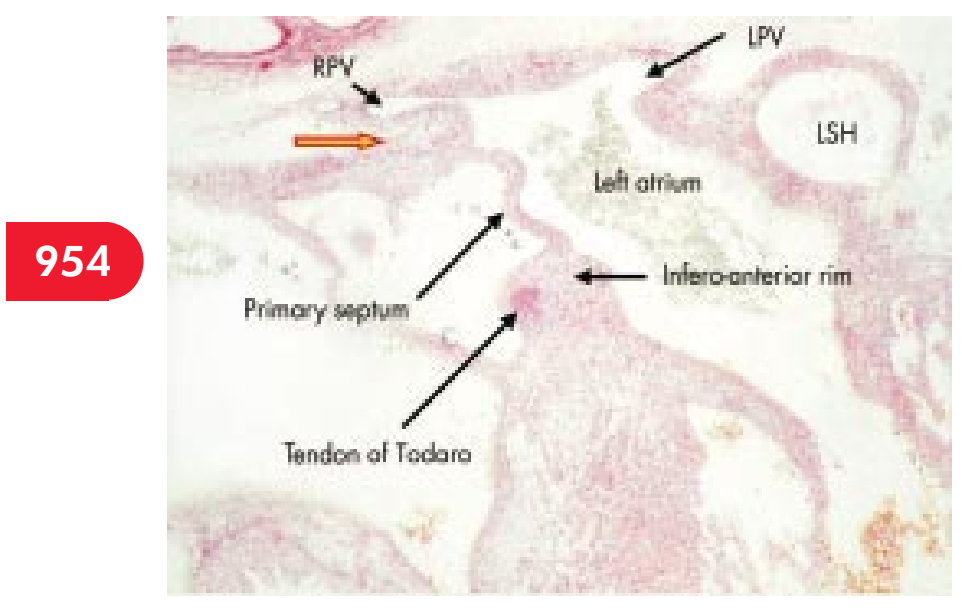

Figure 12 This section is from a human embryo in the eighth week of development, subsequent to the completion of septation. Only at this stage are the right and left pulmonary veins (RPV, LPV) separating and becoming incorporated in the posterior wall of the left atrium. Also only at this stage is there folding of the superior atrial wall (yellow and red arrow) to produce the so-called "septum secundum", in reality part of the interatrial fold. By this stage, the mesenchymal mass formed by fusion of the vestibular spine and mesenchymal cap on the primary atrial septum has muscularised, leaving the central core as the fibrous tendon of Todaro. Note the separate walls of the left sinus horn (LSH).

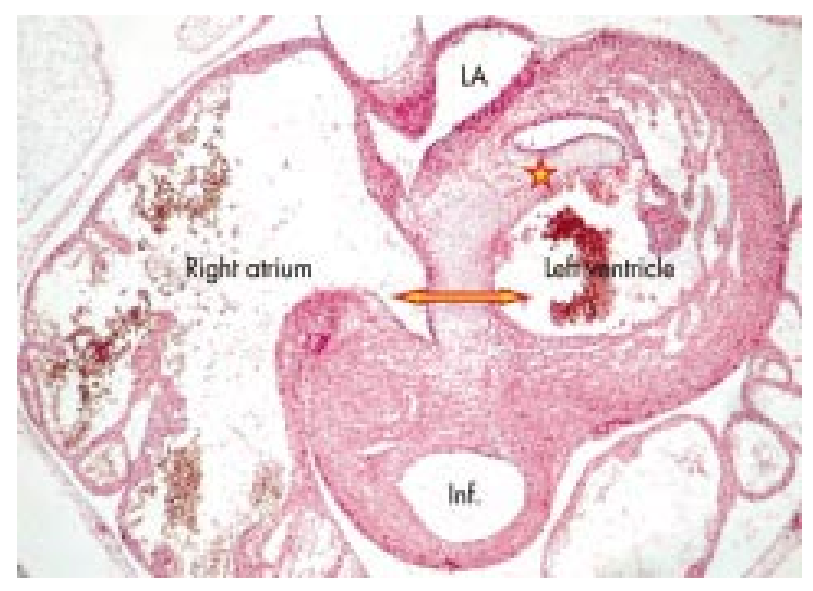

Figure 13 This short axis section is taken from a human heart in the eighth week of development, subsequent to the completion of septation. It shows how the fused atrioventricular cushions have now formed the atrioventricular component of the membranous septum interposed between right atrium and subaortic vestibule (double headed arrow), and also the aortic leaflet of the mitral valve (red and yellow star). Inf., subpulmonary infundibulum; LA, left atrium.

canal on the atrial side of the new junctions, thus forming the vestibules of the two atriums. ${ }^{10}$

The atrioventricular cushions themselves occupy the newly formed septal component of the junctions. As explained above, the greater part of the superior cushion, along with a smaller part of the inferior cushion, are located within the left ventricle, where they form the basis of the aortic leaflet of the mitral valve. ${ }^{11}$ The ventricular aspect of this leaflet will eventually form the posterior part of the vestibule of the subaortic outflow tract (fig 13). The fused part of the cushions attached to the muscular ventricular septum itself, with the muscularising vestibular spine adherent to its atrial aspect, now forms the atrioventricular septal component of the so-called central fibrous body. The other part of this body is the

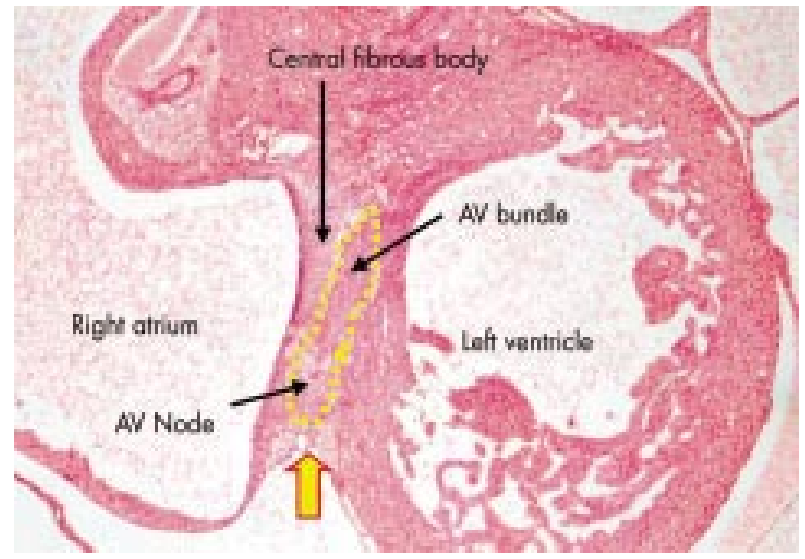

Figure 14 This section, in oblique short axis plane, is from a human embryo at Carnegie stage 22, just subsequent to the completion of septation. It is taken through the newly developed atrioventricular septal area, and has cut through the initial atrioventricular canal musculature that persists as the atrioventricular conduction axis (yellow dotted lines). The superior part of the musculature is now the atrioventricular bundle, with the inferior part becoming sequestrated within the atrial myocardium as the atrial wall folds over the ventricular muscle mass (yellow and red arrow). The fused central parts of the endocardial cushions have now become the central fibrous body.

rightward end of the arch of cushion tissue now occupying the roof of the left ventricle, which will become the right fibrous trigone. The central fibrous body, therefore, represents the fused remnants of the atrioventricular endocardial cushions. This fibrous tissue now occupies the space that, initially, was the central part of the atrioventricular canal. The musculature found immediately beneath and behind the fibrous tissue, therefore, is the musculature of the inferior quadrant of the atrioventricular canal, which is primary myocardium. ${ }^{12}$ This part of the canal musculature retains its continuity with both the atrial and ventricular muscular masses, becoming the atrioventricular node and the penetrating atrioventricular bundle. ${ }^{13}$

At the time that the cushions themselves initially fused to divide the right from the left atrioventricular junctions, this muscular pathway, being the wall of the atrioventricular canal, marked the postero-inferior extent of the cardiac cavities (fig 14). Subsequent to the sequestration of the right side of the initial musculature of the atrioventricular canal into the right atrium, the atrial end of the primary myocardium has become the atrioventricular node (fig 15), positioned in the definitive heart at the apex of the triangle of Koch. The atrial musculature forming the floor of the triangle of Koch has, by now, come to overlie the right ventricular septal musculature that was excavated concomitant with formation of the right ventricular inlet. It is the overlap of the muscular walls in this area that, initially, we described as representing a "muscular atrioventricular septum". ${ }^{4}$ In reality, it is the process of expansion and separation of the atrial and ventricular muscle masses, rather than septation itself, that incorporates the wedge of extracardiac fibro-adipose tissue between the atrial and ventricular walls.

On the ventricular side, it is only subsequent to the closure of the interventricular foramen (see below) that delamination occurs within the myocardium to form the septal leaflet and inferior leaflets of the tricuspid valve, along with their supporting tension apparatus. ${ }^{14}$ Also only subsequent to delamination of these leaflets ${ }^{15}$ is it possible to recognise the ventricular component of the membranous septum (fig 16). This 


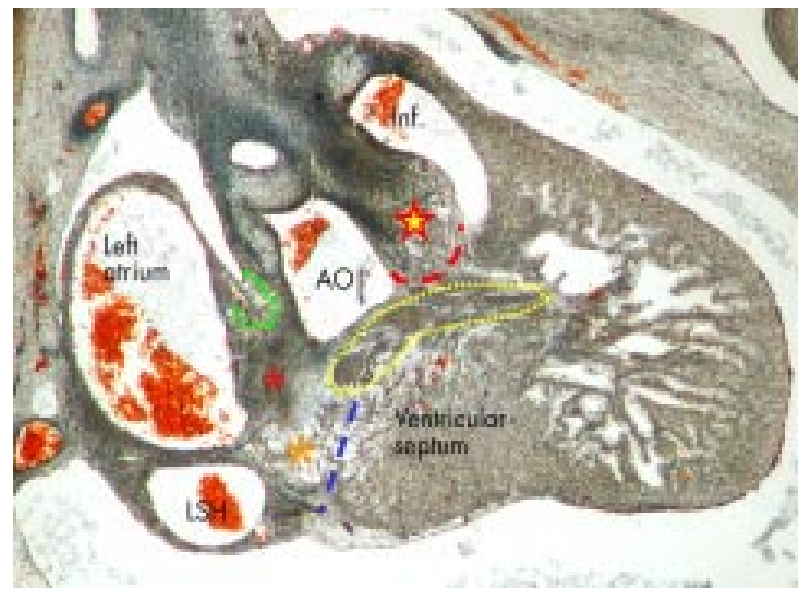

Figure 15 This section, in parasternal long axis plane, is from a human embryo at Carnegie stage 20. Ventricular septation is almost complete, and in this plane, the lower end of the oufflow cushions (star), separating the subaortic vestibule (AO) from the subpulmonary infundibulum (Inf.), has fused with the muscular ventricular septum (red dashed line), the septum carrying on its crest the axis of atrioventricular conduction tissue (yellow dotted lines). In the area of the septal atrioventricular junction, the endocardial cushions have fused to form the central fibrous body (red asterisk), but note that the musculature of the inner heart curve still separates the developing leaflets of the aortic valve from the fibrous tissue (green dashed line). The area of formation of the atrioventricular muscular sandwich is seen inferior to the fibrous body, where the initial musculature of the atrioventricular canal (large orange asterisk) is disappearing in the region of formation of the inferior atrioventricular groove (blue dashed line). Note how the left sinus horn (LSH) is now incorporated within the left atrioventricular groove.

fibrous part of the septum is derived from the most proximal parts of the outflow cushions. ${ }^{16}$ It is the fusion of this ventricular component of the membranous septum with its atrioventricular component, the latter derived from the fused atrioventricular cushions, that closes the embryonic interventricular foramen, thus walling the aorta into the left ventricle (fig 17).

\section{FORMATION OF THE VENTRICULAR SEPTUM}

As explained in our first review, ${ }^{1}$ the muscular part of the ventricular septum is produced concomitant with the ballooning of the apical components of the right and left ventricles from the primary heart tube. As these "pouches" grow out from the heart tube, so the muscular septum is formed between them (fig 18). Until recently, there was disagreement concerning the developmental structures that contributed to the definitive muscular septum. For quite some time, it was believed that the inlet part of the septum formed separately from the primary apical muscular septum. ${ }^{17}$ Indeed, one of us (RHA), in early attempts to explain the development of the heart, illustrated the inlet part of the ventricular septum as a separate structure. ${ }^{18}$ Along these lines, others have argued that there is a muscular septum of the atrioventricular canal incorporated into the definitive ventricular septum. ${ }^{19}$ When adjudicating this process, it should be noted that there are pronounced differences between birds and mammals in the formation of the ventricular septum. In the chick, it is known that the primary apical muscular septum itself compacts by fusion of a series of layered trabeculations. ${ }^{20}$ Also in the chick, it is known that muscularisation of the atrioventricular cushions does, indeed, produce a muscular septum of the atrioventricular canal. ${ }^{21}{ }^{22}$

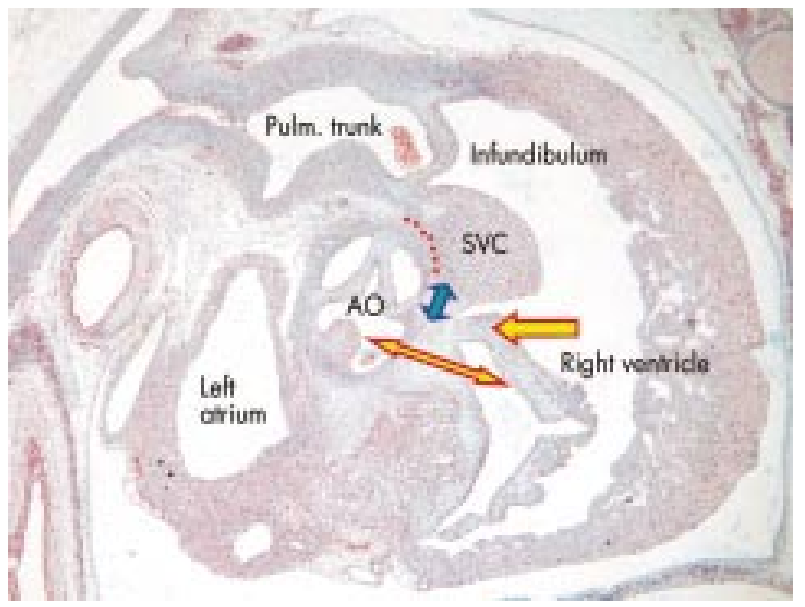

Figure 16 This section, from a human embryo in the eighth week of development, subsequent to the completion of septation, is again sectioned in parasternal long axis plane. It shows that the antero-superior leaflet of the tricuspid valve (red and yellow arrow) has now formed within the cavity of the right ventricle. The central fibrous body, formed by fusion of the atrioventricular cushions, now forms the atrioventricular component of the membranous septum (red and yellow double headed arrow). The interventricular part of the membranous septum (blue and yellow arrow) is developing from the proximal end of the fused oufflow cushions, the remainder of the cushions having muscularised at this stage to form the supraventricular crest (SVC). A tissue plane will form along the line of red dots eventually to separate part of the crest from the aorta $(\mathrm{AO})$ as the free standing subpulmonary infundibulum.

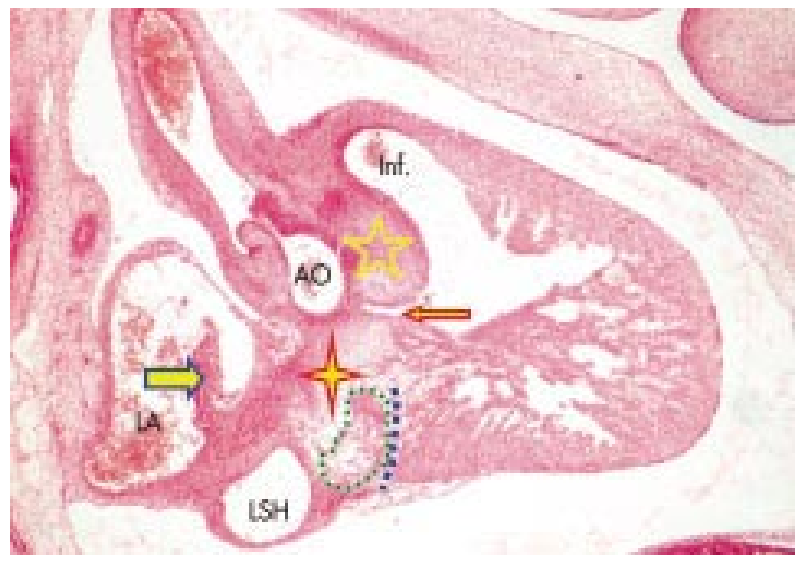

Figure 17 This section, in parasternal long axis plane, is from a human embryo at Carnegie stage 20, just before final closure of the interventricular foramen (red and yellow arrow). The proximal end of the fused oufflow cushions (five pointed star) is dividing the subpulmonary infundibulum (Inf.) from the subaortic vestibule. The oufflow cushions have almost fused with the central fibrous body formed by fusion of the atrioventricular endocardial cushions (four pointed star). Beneath the central fibrous body, the atrioventricular canal musculature (green dotted line) is being sequestrated as the vestibule of the right atrium by formation of the inferior

atrioventricular groove (blue dotted line). The primary atrial septum (blue and yellow arrow) is attached to the atrial aspect of the fibrous body, with the left sinus horn (LSH) forming the coronary sinus in the left atrioventricular groove. Note the maturation of the superior end of the atrioventricular canal musculature to form the atrioventricular node.

Thus far, no one has shown that similar mechanisms occur in the mammalian heart.

For the mammalian heart, it was the serendipitous discovery that an antibody to the nodose ganglion of the chick 


\section{6}

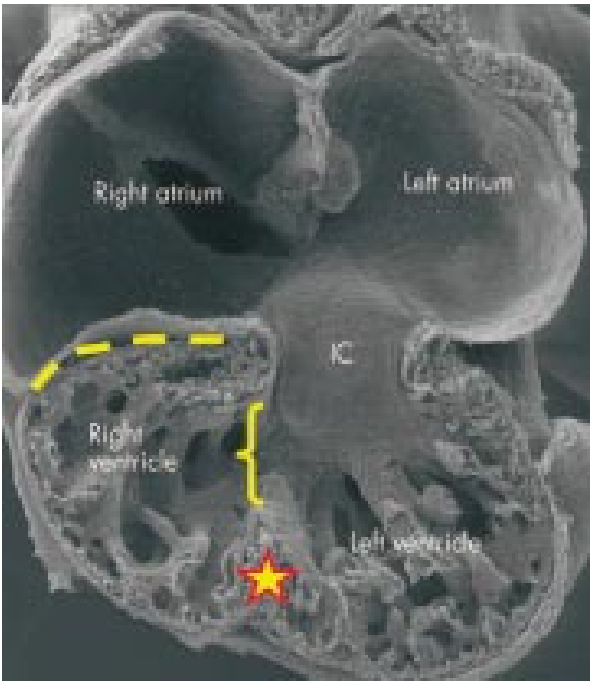

Figure 18 This scanning electron micrograph, from a mouse embryo with 42 somites, shows the formation of the muscular ventricular septum. The specimen was prepared by transecting the heart through the atrioventricular canal, and the photograph is of the posterior segment. Note the inferior cushion (IC) occupying most of the posterior margin of the canal, but note also that the floor of the right atrium is in continuity with the roof of the developing right ventricle at the right margin of the atrioventricular canal, even though the atrioventricular groove interposes between the cavities of right atrium and right ventricle (yellow dashed line). Ballooning of the apical parts of the right and left ventricles from the ascending and descending parts of the ventricular component of the primary heart tube, respectively, has produced the primary muscular ventricular septum between them (star). The primary ventricular foramen (yellow bracket) provides the entrance at this stage to the developing right ventricle.

marked the site of the developing atrioventricular conduction tissues in $\operatorname{man}^{1323}$ that resolved the disputes concerning the structures contributing to the mammalian muscular ventricular septum. These studies ${ }^{132}$ showed unequivocally that the primary muscular ventricular septum produced by ballooning from the primary heart tube separated not only the apical components of the ventricles, but also interposed between the entirety of the inlet of the right ventricle and the cavity of the left ventricle.

Thus, as the apical parts of the ventricles balloon from the primary heart tube, the antibody to the nodose ganglion delimits a band of tissue that surrounds the primary interventricular foramen (fig 19). Inferiorly, the tissue sits on the crest of the newly forming muscular ventricular septum. Superiorly, the tissue lies within the musculature of the atrioventricular canal, marking the site of the subsequent atrioventricular conduction axis. Significantly, the stained tissue, which becomes the conducting tissues, is not only interventricular, but also atrioventricular. Already at this early stage, even before the atrioventricular canal has separated into its right and left components, the cavity of the developing right atrium has direct continuity with that of the forming right ventricle (fig 18). Subsequent to separation of the orifices of the tricuspid and mitral valves, and with expansion of the developing inlet of the right ventricle, formation of the insulating fibro-adipose atrioventricular annulus sequestrates the cardiac muscle positive to the chick nodose ganglion into the distal part of the right atrial musculature (fig 20). The entirety of the definitive right ventricle, therefore, is formed on the ventricular aspect of the positive ring of tissue. The

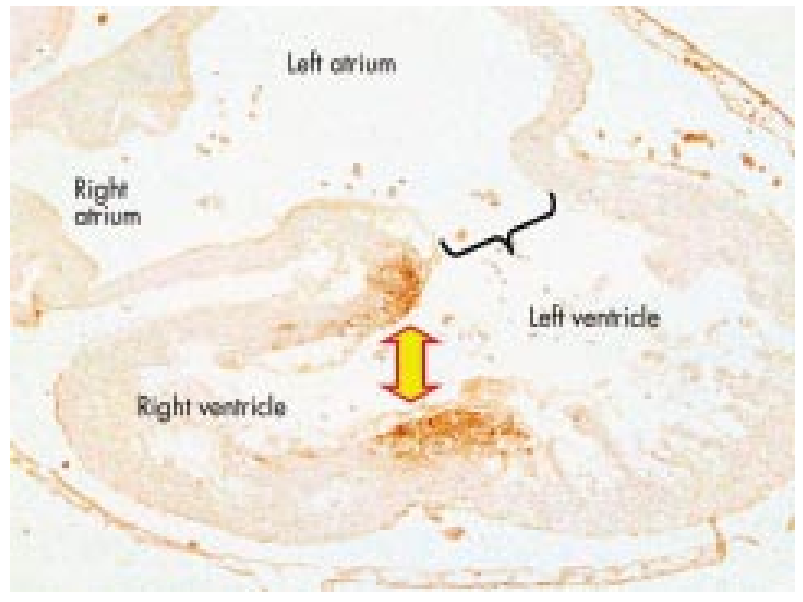

Figure 19 This section from a human embryo at Carnegie stage 14 is comparable to the picture of the mouse heart shown in fig 18 . This section, however, has been stained with an antibody to the nodose ganglion of the chick, which recognises the developing atrioventricular conduction tissues, staining them brown. As can be seen, the conduction tissues at this stage form a ring around the primary interventricular foramen (double headed arrow), the inferior part of the ring marking the crest of the developing muscular ventricular septum, and the superior part occupying the right atrioventricular junction. Thus, at this stage the ring is both interventricular and atrioventricular. The section is taken between the unfused atrioventricular cushions, showing that most of the circumference of the atrioventricular canal is supported by the developing left ventricle (black bracket).

ventricular component of the ring, which forms the atrioventricular bundle, retains its position on the crest of the newly formed ventricular septum. As has already been shown (figs 14 and 15), at the site of its continuity with the atrioventricular node the penetrating bundle is sandwiched between the muscular ventricular septum and the atrioventricular component of the newly formed membranous septum, this now being part of the central fibrous body (fig 13). This anatomic arrangement would not be possible if a second muscular component was incorporated into the definitive ventricular septum, be it derived from a separate muscular "inlet septum" ${ }^{17}$ or a "septum of the atrioventricular canal". ${ }^{19}$ As also explained, such a septum is found within the chick heart, produced by muscularisation of the atrioventricular endocardial cushions. ${ }^{21}{ }^{22}$ In the chick heart, nonetheless, the atrioventricular bundle is buried within the muscular ventricular septum, sandwiched between the primary ventricular septum and the part of the septum derived from the muscularised cushion. ${ }^{24}{ }^{25}$ This arrangement is not seen in the human heart (fig 15). Subsequent to formation of the inlet of the right ventricle, therefore, the primary muscular ventricular septum separates the entirety of the cavities of the ventricles. The additional step then required to permit closure of the embryonic interventricular foramen, separating completely the newly formed right and left ventricles, is the "transfer" of the subaortic outlet to the left ventricle.

Expansion of the atrioventricular canal enlarged the initial continuity that existed between the cavities of the right atrium and right ventricle. Subsequent to this expansion of the canal, the newly formed right ventricle continues to support the entirety of the developing outflow tract. The subaortic outlet component itself has been formed by fusion of the proximal cushions, or ridges, found within the outflow segment of the primary heart tube (fig 15). This embryonic 


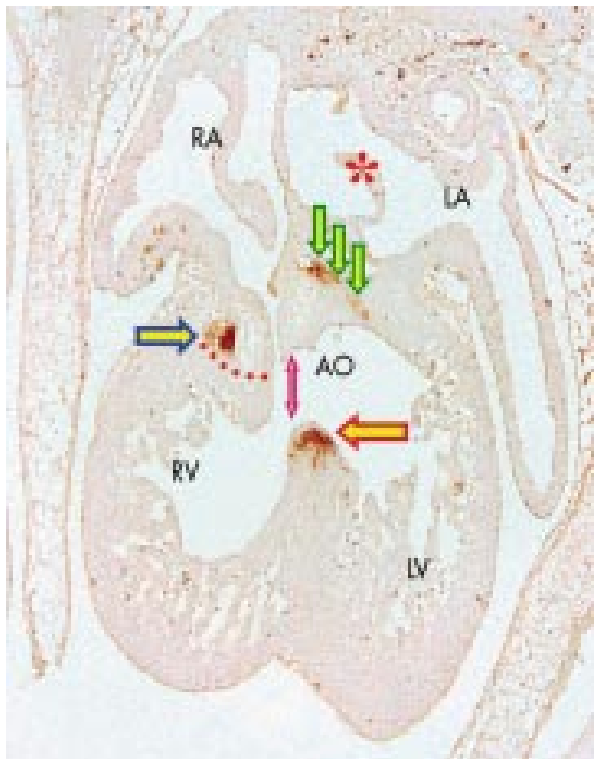

Figure 20 This section, in comparable plane to fig 19, is from a human embryo at stage 20 , again stained with the antibody to the nodose ganglion of the chick. The atrial septum (red asterisk) has now fused with the central fibrous body, dividing right and left atriums (RA, LA). Expansion of the right atrioventricular junction has proceeded concomitant with formation of the inlet of the right ventricle (RV). The ring of atrioventricular conduction tissue is still placed on the crest of the ventricular septum (red and yellow arrow), but the expanded right part of the ring (blue and yellow arrow) is now becoming sequestrated in the vestibule of the right atrium by formation of the parietal right atrioventricular junction (red dotted line). This shows that the entirety of the right ventricle is formed from the tissues initially distal to the embryonic interventricular foramen. The section also shows that the superior part of the ring has been transferred to the left ventricle (LV) as the subaortic vestibule (AO, purple arrows). This will be committed to the left ventricle by eventual fusion of the atrioventricular and oufflow cushions along the plane of the double headed purple and yellow arrow, converting the initial embryonic interventricular foramen into the subaortic vestibule.

outlet septum, when first formed, is of necessity exclusively a right ventricular structure, since the right ventricle at this stage supports the entirety of the developing outflow tract. At this stage, the lumen of the developing aorta is in communication with the left ventricle through the embryonic interventricular foramen, but also remains in continuity with the cavity of the developing right ventricle. It is the fusion of the proximal ends of the outflow cushions with the crest of the muscular ventricular septum that walls the aorta into the left ventricle, this process incorporating the embryonic interventricular communication as the subaortic vestibule (figs 15 and 17). Subsequent to the fusion of the atrioventricular and outflow cushions, the ring of tissue positive to the nodose ganglion of the chick can still be seen as a ring surrounding the subaortic vestibule, showing that not only the pulmonary trunk, but also the aorta, was originally supported by the developing right ventricle (fig 20).

After closure of the embryonic interventricular foramen, the fused outflow cushions, which initially formed an embryonic outlet septum, become transformed into the freestanding muscular subpulmonary infundibulum and supraventricular crest of the right ventricle (fig 16). The most proximal part of the fused outflow cushions, nonetheless, have fused with the atrioventricular cushions to close the embryonic interventricular communication, a process well described by Odgers. ${ }^{16}$ It is the conversion of this part of the fused outflow cushions into fibrous tissue, occurring concomitant with delamination of the leaflets of the tricuspid valve, which produces the interventricular part of the membranous septum, by now an integral part of the subaortic vestibule. ${ }^{15}$ As has been explained, this interventricular part of the membranous septum is separate from, but contiguous with, the atrioventricular part of the membranous septum, the latter being derived from the fused atrioventricular cushions (fig 16).

\section{CONCLUSIONS}

The definitive structures that separate the atrial and ventricular cavities are almost entirely muscular structures, albeit that parts are formed by folds or sandwiches between adjacent walls. The small part of the septum that is not muscular, the membranous septum, is formed in part from the atrioventricular cushions, and in part from the proximal end of the fused outflow cushions. ${ }^{8}{ }^{16}$ Although these outflow cushions at first formed an extensive embryonic outlet septum, this tissue, subsequent to its muscularisation, becomes incorporated into the right ventricle as the supraventricular crest and the free-standing subpulmonary infundibulum. The steps involved in this process, along with formation of the arterial valvar leaflets and sinuses, and development of the intrapericardial parts of the arterial trunks, will be the subjects of our final review of cardiac development.

\section{ACKNOWLEDGEMENTS}

The work done by Professors Anderson and Brown, and Dr Webb, during the preparation of the concepts set out in this review, was supported generously by the British Heart Foundation. The work of Professors Lamers and Moorman was equally well supported by the Dutch Heart Foundation.

\section{...................}

Authors' affiliations

R H Anderson, Institute of Child Health, University College, London, UK S Webb, N A Brown, Department of Anatomy and Developmental Biology, St George's Hospital Medical School, London, UK A Moorman, W Lamers, Academic Medical Center, Amsterdam, The Netherlands

\section{REFERENCES}

1 Moorman AFM, Webb S, Brown NA, et al. The development of the heart: (1) Formation of the cardiac chambers and arterial trunks. Heart 2003;89:806-14.

- In this, the first of our reviews, we discussed the steps involved in formation of the heart tube and the components of the cardiac chambers and arterial trunks.

2 Larsen WJ. Human embryology. New York: Churchill Livingstone $1993 ; 147$

- This otherwise excellent student textbook of embryology used the "classical" account for development of the atrial septum, with purported primary and secondary septums illustrated as overlapping sheets that have grown into the heart.

3 Anderson RH, Brown NA. The anatomy of the heart revisited. Anat Rec 1996;62:710-16.

- The review in which we questioned the definition of septal structures, arguing that it should be possible to resect a "true" septum without exiting from the cavities of the heart.

4 Becker AE, Anderson RH. Atrioventricular septal defects. What's in a name? J Thorac Cardiovasc Surg 1982;83:461-9.

- This review has proved seminal in the classification of hearts with common atrioventricular junction. In retrospect, however, we were wrong when we argued that, in addition to the fibrous atrioventricular septum, there was also a muscular septum. We now know that the purported muscular septum is, in reality, a layer of extracardiac fibroadipose tissue sandwiched between the atrial and ventricular walls.

5 Webb S, Kanani M, Anderson RH, et al. Development of the human pulmonary vein and its incorporation in the morphologically left atrium. Cardiol Young 2001;11:632-42.

- In this study, we showed how the pulmonary vein canalised as a new structure in the medistinal tissues, using the dorsal mesocardium to gain entrance to the primary atrial compartment of 
the heart tube. Only much later are the walls of the separate veins incorporated to form the dome of the left atrium.

6 Anderson RH, Brown NA, Webb S. The development and structure of the atrial septum. Heart 2002;88:104-10.

- In this earlier contribution to Education in Heart, we discussed in greater detail much of the information presented in this review concerning atrial septation.

7 Franco D, Campione M, Kelly R, et al. Multiple transcriptional domains, with distinct left and right components, in the atrial chambers of the

958 - This important study showed how it was possible to distinguish between the molecularly right and left sides of the developing atriums according to the expression of various genes and proteins. The molecular arrangement did not correspond exactly with morphological boundaries.

8 Webb S, Brown NA, Anderson RH. Formation of the atrioventricular septal structures in the normal mouse. Circ Res 1998;82:645-56.

- The study in which we confirmed that far more developmental primordiums were involved in separation of the right and left sides of the atrioventricular canal than mere fusion of the atrioventricular endocardial cushions.

9 His W. Die Area Interposita, die Eustachi'sche Klappe und die Spina vestibuli. Anatomie menslicher embryonen. Leipzig: von F.C.W. Vogel, Leipzig, 1880;149-52

- An exquisite study in which Wilhelm His the elder illustrated the mechanism of development of the heart, and pointed to the crucial role of the "spina vestibuli", a structure subsequently ignored until very recently.

10 Wessels A, Markman MWM, Vermeulen JLM, et al. The development of the atrioventricular junction in human heart. Circ Res 1996:78:1 10-17.

- The study that showed how, subsequent to formation of the fibro-adipose atrioventricular junctions, the initial musculature of the embryonic atrioventricular canal was sequestrated on the atrial sides of the new region of electrical insulation.

11 Kim J-S, Virágh S, Moorman AFM, et al. Development of the myocardium of the atrioventricular canal and the vestibular spine in the human heart. Circ Res 2001;88:395-402.

- This study used graded series of human embryos, along with reconstructions, to show the extensive moulding of the atrioventricular cushions occurring during human development as they form the aortic leaflet of the mitral valve. The study also re-emphasised the role of His's "spina vestibuli".

12 Houweling AC, Somi S, Van Den Hoff M, et al. Developmental pattern of ANF gene expression reveals a strict localization of cardiac chamber formation in chicken. Anat Rec 2002:266:93-102.

- This study showed how the distribution of atrial natriuretic peptide distinguished between the areas of the cardiac chambers produced by "ballooning" as opposed to the parts derived from the initial primary heart tube.

13 Wessels A Vermeulen JLM, Verbeek FJ, et al Spatial distribution of "tissue-specific" antigens in the developing human heart and skeletal muscle. III. An immunohistochemical analysis of the distribution of the neural tissue antigen G1N2 in the embryonic heart; implications for the development of the atrioventricular conduction system. Anat Rec 1992:232:97-111.

- The initial study that demonstrated how the axis of atrioventricular conduction tissue was derived from a ring of tissue encircling the primary interventricular canal, but a ring that also, from the outset, occupied part of the right atrioventricular junction.

14 Lamers WH, Virágh S, Wessels A, et al. Formation of the tricuspid valve in the human heart. Circulation 1995;91:111-21.

- This study charted the expansion of the right atrioventricular junction in the human heart, distinguishing those leaflets of the tricuspid valve involving the various cushions, and stressing the late delamination of parts of the leaflets from the ventricular myocardium.
15 Allwork SP, Anderson RH. Developmental anatomy of the membranous part of the ventricular septum in the human heart. Br Heart J 1979;41:275-80.

- This study, performed much earlier than the previous one, also stressed the role of late delamination of the leaflets of the tricuspid valve in formation of the membranous part of the septum.

16 Odgers PNB. The development of the pars membranacea septi in the human heart. J Anat 1938;72:247-59.

- A detailed study, difficult to understand, but showing that the endocardial cushions of both the atrioventricular canal and the outflow tract contributed to formation of the membranous part of the septum.

17 Wenink ACG. Embryology of the ventricular septum. Separate origin of its components. Virchows Archives A: Pathological Anatomy and Histology 1981;390:71-9.

18 Anderson RH, Becker AE. Cardiac anatomy. An integrated text and colour atlas. London: Gower Medical Publishing, 1981;10.1-10.30.

19 Van Praagh R, Geva T, Kreutzer J. Ventricular septal defects: how shal we describe, name and classify them? J Am Coll Cardiol $1989 ; 14: 1298-9$.

- In all three of these studies, it was presumed that the muscular part of the ventricular septum was derived from more than one embryonic primordium. As we have now shown, there is no convincing evidence that supports this notion for development of the mammalian heart.

20 Ben-Shachar G, Arcilla RA, Lucas RV, et al. Ventricular trabeculations in the chick embryo heart and their contribution to ventricular and muscular septal development. Circ Res 1985;57:759-66.

- This exquisite study demonstrated convincingly that, in the chick, the ventricular septum was formed by the fusion of multiple layered trabeculations. There is no evidence thus far that the mammalian septum develops in comparable fashion.

21 de la Cruz MV, Gimenez-Ribotta M, Saravalli O, et al. The contribution of the inferior endocardial cushion of the atrioventricular canal to cardiac septation and to the development of the atrioventricular valves: study in the chick embryo. Am J Anat 1983;166:63-72.

- By using marking with Indian ink, these investigators showed that, in the chick, the crest of the muscular ventricular septum was derived from the atrioventricular endocardial cushions. Again, there is no evidence to show that the mammalian septum develops in comparable fashion.

22 van den Hoff MJB, Kruithof BPT, Moorman AFM, et al. Formation of myocardium after the initial development of the linear heart tube. Dev Biol 2001;240:61-76.

- This study, using molecular techniques, confirmed that, in the chick, there is muscularisation of the atrioventricular endocardial cushions, the newly formed muscular area becoming incorporated within the ventricular septum.

23 Lamers WH, Wessels A, Verbeek FJ, et al. New findings concerning ventricular septation in the human heart. Implications for maldevelopment. Circulation 1992;86: 1194-205.

- The study that amplified the findings initially described in reference 12 , relating the findings to tricuspid atresia and double inlet left ventricle.

24 Davies F. The conducting system of the bird's heart. J Anat 1930;64:129-46.

25 Vassall-Adams PR. The developing atrioventricular bundle and its branches in relation to small induced ventricular septal defects in the heart of chick embryos. J Anat 1982;134:209-14.

- These studies showed how, in the bird heart, the atrioventricular bundle is sandwiched within the musculature of the ventricular septum, a finding consistent with the observation that the crest of the septum is formed by muscularisation of the atrioventricular endocardial cushions. 